\title{
A REVERSE HÖLDER TYPE INEQUALITY FOR THE LOGARITHMIC MEAN AND GENERALIZATIONS
}

\author{
JOHN MALONEY ${ }^{1}$, JACK HEIDEL $^{1}$ and JOSIP PEC̆ARIĆ ${ }^{2}$
}

(Received 4 October 1995; revised 6 July 1998)

\begin{abstract}
An inequality involving the logarithmic mean is established. Specifically, we show that

$$
L(c, x)^{\frac{\ln (c / x)}{\ln (c / a)}} L(x, a)^{\frac{\ln (x / a)}{\ln (c / a)}}<L(c, a),
$$

where $0<a<x<c$ and $L(x, y)=\frac{y-x}{\ln x-\ln y}, 0<x<y$. Then several generalizations are given.
\end{abstract}

\section{Introduction}

The logarithmic mean,

$$
L(x, y)=\frac{y-x}{\ln x-\ln y}, \quad 0<x<y
$$

has many applications in statistics and economics [9]. It is well known, and easily established $[1,3,7,10]$ that

$$
G(x, y) \leq L(y, x) \leq A(y, x)
$$

where $G(y, x)=\sqrt{x y}$ is the geometric mean and $A(x, y)=(x+y) / 2$ is the arithmetic mean. In fact, writing $A(x, y)=M_{1}(y, x)$, where

$$
M_{p}(y, x)=\left(\frac{y^{p}+x^{p}}{2}\right)^{1 / p},
$$

${ }^{1}$ Department of Mathematics, University of Nebraska at Omaha, Omaha Nebraska 68182, USA

${ }^{2}$ Faculty of Textile Technology, Zagreb Croatia

(C) Australian Mathematical Society 2000, Serial-fee code 0334-2700/00 
it is known [7] that $M_{p_{1}}(y, x) \leq M_{p_{2}}(y, x)$ for $p_{1} \leq p_{2}$. It is also known [4-6,9,12] that

$$
L(y, x) \leq M_{1 / 3}(y, x) .
$$

On the other hand, Hölder's inequality states that

$$
M_{1}\left(y_{1} y_{2}, x_{1} x_{2}\right) \leq M_{p}\left(y_{1}, x_{1}\right) M_{q}\left(y_{2}, x_{2}\right),
$$

if $1 / p+1 / q=1$ with $p, q>0$. It is thus curious that the logarithmic mean $L(y, x)$ satisfies the inequality

$$
L(c, x)^{\frac{\ln (c / x)}{\ln _{(c / a)}}} L(x, a)^{\frac{\ln (x / a)}{\ln (c / a)}}<L(c, a),
$$

where $0<a<x<c$ and it is noted that

$$
\frac{\ln (c / x)}{\ln (c / a)}+\frac{\ln (x / a)}{\ln (c / a)}=1
$$

It is the reverse Hölder type inequality (1) which is the subject of this note and will be established below. Relation (1) arises in a parameter identification problem for a fractal Michaelis-Mention equation [8].

In the following, use will be made of Jensen's inequality [11] which we now state for the reader's convenience.

JENSEN'S INEQUALITY. If ,

(1) $w_{i}>0 \forall i=1,2, \ldots, n$,

(2) $\alpha_{0}, \alpha_{1}, \alpha_{2}, \ldots, \alpha_{n} \in R$,

(3) $\Phi:[0, \infty) \rightarrow R$ is a strictly convex function,

then

$$
\left(\sum_{i=1}^{n} w_{i}\right) \Phi\left(\frac{\sum_{i=1}^{n} w_{i} \alpha_{i}}{\sum_{i=1}^{n} w_{i}}\right) \leq \sum_{i=1}^{n} w_{i} \Phi\left(\alpha_{i}\right)
$$

and the inequality is strict unless $\alpha_{0}=\alpha_{1}=\alpha_{2}=\cdots=\alpha_{n}$.

\section{Main result}

LEMMA 2.1. Let $g(u)=\frac{\ln u}{u-1}$, where $g(1)=1$. Then for all $u>0$

(i) $g$ is a strictly decreasing function of $u$,

(ii) $\lim _{u \rightarrow 0^{+}} g(u)=\infty, \lim _{u \rightarrow \infty} g(u)=0, \lim _{u \rightarrow 1} g(u)=1$,

(iii) $g(1 / u)=u g(u)$. 
PROOF.

$$
g^{\prime}(u)=\frac{1-(1 / u)-\ln u}{(1-u)^{2}} .
$$

Set $z(u)=1-(1 / u)-\ln u$. Then $z^{\prime}(u)=(1 / u)(1 / u-1)$ which is positive for $0<u<1$ and negative for $u>1$. Thus $z(u)$ increases from $-\infty$ at $u=0$ to 0 at $u=1$ and then decreases to $-\infty$ as $u$ tends to $\infty$. Thus $g^{\prime}(u)$ is negative except at $u=1$. This establishes (i). The limits in (ii) can be computed in the usual fashion using L'Hôpital's rule. For (iii) we have

$$
g(1 / u)=\frac{\ln (1 / u)}{1 / u-1}=u g(u) .
$$

LEMMA 2.2. Let $f(x)=x-\ln x$. Then

(i) $f$ is decreasing on $(0,1)$ and increasing on $(1, \infty)$,

(ii) $\lim _{x \rightarrow 0^{+}} f(x)=\infty, f(1)=1$ and $\lim _{x \rightarrow \infty} f(x)=\infty$,

(iii) if $\alpha>0, x>0$ then $f(\alpha x)=f(x)$ for $x=g(\alpha)$ so that $f(\alpha g(\alpha))=$ $f(g(\alpha))$

Proof. Parts (i) and (ii) can be established in the usual way. For (iii) we have

$$
f(\alpha x)=f(x) \Rightarrow \alpha x-\ln (\alpha x)=x-\ln x \Rightarrow(\alpha-1) x=\ln \alpha \Rightarrow x=g(\alpha) .
$$

Let $y(x)$ denote the left-hand side of (1), and set $\alpha=\ln c-\ln a$. Note that $y(x)>0$ $\forall a<x<c$. Then

$$
\begin{aligned}
\alpha \ln y= & {[\ln c-\ln x][\ln (c-x)-\ln (\ln c-\ln x)] } \\
& +[\ln x-\ln a][\ln (x-a)-\ln (\ln x-\ln a)]
\end{aligned}
$$

and so

$$
\begin{aligned}
\frac{\alpha y^{\prime}}{y}= & -\frac{1}{x}[\ln (c-x)-\ln (\ln c-\ln x)]+[\ln c-\ln x]\left[\frac{-1}{c-x}-\frac{-1 / x}{\ln c-\ln x}\right] \\
& +\frac{1}{x}[\ln (x-a)-\ln (\ln x-\ln a)]+[\ln x-\ln a]\left[\frac{1}{x-a}-\frac{1 / x}{\ln x-\ln a}\right] \\
= & \frac{1}{x}\left[\ln \left[\frac{x-a}{\ln x-\ln a}\right]\right]+\left[\frac{\ln x-\ln a}{x-a}\right]-\frac{1}{x} \\
& +\frac{1}{x}-\frac{\ln c-\ln x}{c-x}-\frac{1}{x} \ln \left[\frac{c-x}{\ln c-\ln x}\right] \\
= & \frac{1}{x} \ln \left[x \frac{a / x-1}{\ln (a / x)}\right]+\frac{1}{x} \frac{\ln (a / x)}{a / x-1}-\frac{1}{x} \frac{\ln (c / x)}{c / x-1}-\frac{1}{x} \ln \left[x \frac{c / x-1}{\ln (c / x)}\right] \\
= & \frac{1}{x}[f(g(a / x))-f(g(c / x))]=\frac{1}{x} h(x) .
\end{aligned}
$$


Now $f(g(a / x))$ is an increasing function of $x$ while $f(g(c / x))$ is a decreasing function of $x$ so that $h(x)$ is an increasing function of $x$. Clearly $\alpha y^{\prime} / y$ is zero at exactly one point which implies that $y^{\prime}$ is zero at exactly one point.

LEMMA 2.3. $y^{\prime}$ is zero at the point $x=\sqrt{a c}$.

PROOF. Now $f(g(c / x))=f(g(a / x))=f\left(\frac{a}{x} g(a / x)\right)$, from Lemma 2.3 (iii), so that $g(c / x)=(a / x) g(a / x)=g(x / a)$ by Lemma 2.2 (iii). Thus $c / x=x / a$ which gives $x=\sqrt{a c}$.

THEOREM 2.4. For all values of $0<a<x<c$

$$
\left(\frac{c-x}{\ln c-\ln x}\right)^{\ln c-\ln x}\left(\frac{x-a}{\ln x-\ln a}\right)^{\ln x-\ln a}<\left(\frac{c-a}{\ln c-\ln a}\right)^{\ln c-\ln a} .
$$

PROOF. The result holds if and only if

$$
\begin{aligned}
(\ln c- & \ln x) \ln \left(\frac{c-x}{\ln c-\ln x}\right)+(\ln x-\ln a) \ln \left(\frac{x-a}{\ln x-\ln a}\right) \\
& <(\ln c-\ln a) \ln \left(\frac{c-a}{\ln c-\ln a}\right) .
\end{aligned}
$$

Setting $x_{0}=a, x_{1}=x, x_{2}=c$ and letting $w_{i}=\ln x_{i}-\ln x_{i-1}, \alpha_{i}=\frac{x_{i}-x_{i-1}}{\ln x_{i}-\ln x_{i-1}}$ and $\Phi(x)=-\ln x$, the result follows from the Jensen's inequality with $\leq$ rather than $<$.

But

$$
\alpha y^{\prime}=\frac{y}{x}[f(g(a / x))-f(g(c / x))]
$$

so that $y^{\prime}$ is negative on $[a, \sqrt{a c}]$ and positive on $[\sqrt{a c}, c]$. Strict inequality in Theorem 2.4 now follows from the previous results since the derivative is strictly negative on $[a, \sqrt{a c}]$ and positive on the interval $[\sqrt{a c}, c]$. Thus equality holds only at $a$ and $c$.

\section{Convexity}

THEOREM 3.1. The function

$$
y(x)=\left(\frac{c-x}{\ln c-\ln x}\right)^{\frac{\ln c-\ln x}{\ln c-\ln a}}\left(\frac{x-a}{\ln x-\ln a}\right)^{\frac{\ln x-\ln a}{\ln c-\ln a}}
$$

is log-convex, and hence convex, on the interval $[a, \sqrt{a c}]$. 
PROOF. Let $w=\alpha \ln y$; then $w^{\prime}=\alpha y^{\prime} / y$ and hence from (2) $x w^{\prime}=f(g(a / x))-$ $f(g(c / x))$ is an increasing function so that $w^{\prime}+x w^{\prime \prime} \geq 0$. Thus $x w^{\prime \prime} \geq-w^{\prime}$. Now on $[a, \sqrt{a c}], w^{\prime} \leq 0$ and so $w^{\prime \prime} \geq 0$ so that $w$ is convex (and hence log-convex) on the interval $[a, \sqrt{a c}]$.

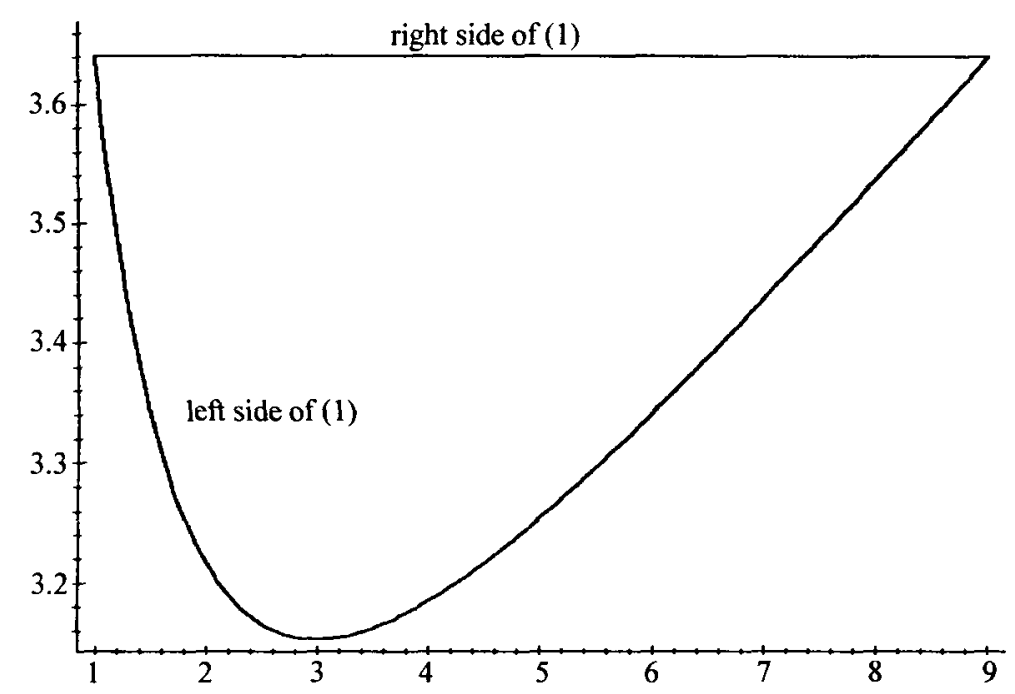

FigURE 1. Graph of equation (1) with $a=1, c=9$.

Figure 1 indicates that the function is also probably convex on the interval $[\sqrt{a c}, c]$. However we have not been able to establish this even with the aid of the next result.

LEMMA 3.2. The curve

$$
y(x)=\left(\frac{c-x}{\ln c-\ln x}\right)^{\frac{\ln c-\ln x}{\ln c-\ln a}}\left(\frac{x-a}{\ln x-\ln a}\right)^{\frac{\ln x-\ln a}{\ln c-\ln a}}
$$

is invariant under the transformation $x \rightarrow a c / x$.

PROOF.

$$
\begin{aligned}
z(x) & =\left(\frac{c-a c / x}{\ln c-\ln (a c / x)}\right)^{\frac{\ln c-\ln (a c / x)}{\ln c-\ln a}}\left(\frac{a c / x-a}{\ln (a c / x)-\ln a}\right)^{\frac{\ln (a c / x)-\ln a}{\ln c-\ln a}} \\
& =\left(\frac{c(x-a) / x}{\ln c-\ln a-\ln c+\ln x}\right)^{\frac{\ln c-\ln (a c)+\ln x}{\ln c-\ln a}}\left(\frac{a(c-x) / x}{\ln (a c)-\ln x-\ln a}\right)^{\frac{\ln (a c c)-\ln x-\ln a}{\ln c-\ln a}} \\
& =\left(\frac{c(x-a) / x}{\ln x-\ln a}\right)^{\frac{\ln x-\ln a}{\ln c-\ln a}}\left(\frac{a(c-x) / x}{\ln c-\ln x}\right)^{\frac{\ln c-\ln x}{\ln c-\ln a}} \\
& =(c / x)^{\frac{\ln x-\ln a}{\ln a-\ln a}}(a / x)^{\frac{\ln c-\ln x}{\ln c-\ln a}} y(x)
\end{aligned}
$$


Now,

$$
\frac{\ln x-\ln a}{\ln c-\ln a}+\frac{\ln c-\ln x}{\ln c-\ln a}=1
$$

Thus, from (4)

$$
\begin{aligned}
(c / x)^{\frac{\ln x-\ln a}{\ln c-\ln a}}(a / x)^{\frac{\ln c-\ln x}{\ln c-\ln a}} & =(c / x)^{\frac{\ln x-\ln a}{\ln c-\ln a}}(a / x)^{1-\frac{\ln x-\ln a}{\ln c-\ln a}} \\
& =\frac{(c / x)^{\frac{\ln x-\ln a}{\ln c-\ln a}}}{(a / x)^{\frac{\ln x-\ln a}{\ln c-\ln a}} \frac{a}{x}} \\
& =(a / x)(c / a)^{\ln (x / a) / \ln (c / a)} \\
& =\frac{a}{x} \frac{x}{a}=1 \quad \text { since } b^{x}=e^{x \ln b}
\end{aligned}
$$

Thus $z(x)=y(x)$ and the lemma is proved.

\section{Generalizations and applications}

The following theorems follow directly from Jensen's inequality and are generalizations of Theorem 2.1 .

THEOREM 4.1. If

(1) $\Phi:[0, \infty) \rightarrow R$ is a function,

(2) $f, g:[0, \infty) \rightarrow R$ are increasing functions,

(3) $A_{0} \leq A_{1} \leq \cdots \leq A_{n}$,

then

(1) if $\Phi$ is convex then

$$
\begin{aligned}
\left(g\left(A_{n}\right)\right. & \left.-g\left(A_{0}\right)\right) \Phi\left(\frac{f\left(A_{n}\right)-f\left(A_{0}\right)}{g\left(A_{n}\right)-g\left(A_{0}\right)}\right) \\
& \leq \sum_{i=1}^{n}\left(g\left(A_{i}\right)-g\left(A_{i-1}\right)\right) \Phi\left(\frac{f\left(A_{i}\right)-f\left(A_{i-1}\right)}{g\left(A_{i}\right)-g\left(A_{i-1}\right)}\right),
\end{aligned}
$$

(2) if $\Phi$ is concave then

$$
\begin{aligned}
\left(g\left(A_{n}\right)\right. & \left.-g\left(A_{0}\right)\right) \Phi\left(\frac{f\left(A_{n}\right)-f\left(A_{0}\right)}{g\left(A_{n}\right)-g\left(A_{0}\right)}\right) \\
& \geq \sum_{i=1}^{n}\left(g\left(A_{i}\right)-g\left(A_{i-1}\right)\right) \Phi\left(\frac{f\left(A_{i}\right)-f\left(A_{i-1}\right)}{g\left(A_{i}\right)-g\left(A_{i-1}\right)}\right),
\end{aligned}
$$


(3) if $\Phi$ is log-convex then

$$
\Phi\left(\frac{f\left(A_{n}\right)-f\left(A_{0}\right)}{g\left(A_{n}\right)-g\left(A_{0}\right)}\right)^{\left(g\left(A_{n}\right)-g\left(A_{0}\right)\right)} \leq \prod_{i=1}^{n} \Phi\left(\frac{f\left(A_{i}\right)-f\left(A_{i-1}\right)}{g\left(A_{i}\right)-g\left(A_{i-1}\right)}\right)^{\left(g\left(A_{i}\right)-g\left(A_{i-1}\right)\right)},
$$

(4) if $\Phi$ is log-concave then

$$
\Phi\left(\frac{f\left(A_{n}\right)-f\left(A_{0}\right)}{g\left(A_{n}\right)-g\left(A_{0}\right)}\right)^{\left(g\left(A_{n}\right)-g\left(A_{0}\right)\right)} \geq \prod_{i=1}^{n} \Phi\left(\frac{f\left(A_{i}\right)-f\left(A_{i-1}\right)}{g\left(A_{i}\right)-g\left(A_{i-1}\right)}\right)^{\left(g\left(A_{i}\right)-g\left(A_{i-1}\right)\right)} .
$$

Proof. In Jensen's inequality set $w_{i}=g\left(A_{i}\right)-g\left(A_{i-1}\right)$ and $\alpha_{i}=\frac{f\left(A_{i}\right)-f\left(A_{i-1}\right)}{g\left(A_{i}\right)-g\left(A_{i-1}\right)}$ and the result follows.

As a first application let $M, N: R \rightarrow R$ be differentiable functions with $N$ strictly monotone. Given any two numbers $a$ and $b$, there is a number $c$, according to the mean value theorem, such that

$$
\frac{M(b)-M(a)}{N(b)-N(a)}=\frac{M^{\prime}(c)}{N^{\prime}(c)}
$$

for some $c, a<c<b$. If $c$ is uniquely determined then it is called the (M.N) mean-value mean of $a$ and $b$ [2]. In this case let $H$ be the inverse of $M^{\prime} / N^{\prime}$ and write

$$
c=H\left(\frac{M(b)-M(a)}{N(b)-N(a)}\right) .
$$

If $M$ and $N$ are both increasing and $H$ is either log-convex or log-concave, we can apply one of the inequalities in Theorem 4.1 to write

$$
H\left(\frac{M\left(A_{n}\right)-M\left(A_{0}\right)}{N\left(A_{n}\right)-N\left(A_{0}\right)}\right) \leq \prod_{i=1}^{n} H\left(\frac{M\left(A_{i}\right)-M\left(A_{i-1}\right)}{N\left(A_{i}\right)-N\left(A_{i-1}\right)}\right)^{\frac{N\left(A_{i}\right)-N\left(A_{i-1}\right)}{N\left(A_{n}\right)-N\left(A_{0}\right)}},
$$

or

$$
H\left(\frac{M\left(A_{n}\right)-M\left(A_{0}\right)}{N\left(A_{n}\right)-N\left(A_{0}\right)}\right) \geq \prod_{i=1}^{n} H\left(\frac{M\left(A_{i}\right)-M\left(A_{i-1}\right)}{N\left(A_{i}\right)-N\left(A_{i-1}\right)}\right)^{\frac{N\left(A_{i}\right)-N\left(A_{i-1}\right)}{N\left(A_{n}\right)-N\left(A_{0}\right)}},
$$

where we have made the associations that $\Phi=h, f=M, g=N, A_{n}=b, A_{0}=a$.

Now specializing to the case of $\Phi(x)=x(\log$-concave $\Phi)$ in Theorem 4.1 we obtain

$$
\frac{f\left(A_{n}\right)-f\left(A_{0}\right)}{g\left(A_{n}\right)-g\left(A_{0}\right)} \geq \prod_{i=1}^{n}\left(\frac{f\left(A_{i}\right)-f\left(A_{i-1}\right)}{g\left(A_{i}\right)-g\left(A_{i-1}\right)}\right)^{\frac{g\left(A_{i}\right)-g\left(A_{i-1}\right)}{g\left(A_{n}\right)-g\left(A_{0}\right)}}
$$


and interchanging $f$ and $g$ we can write

$$
\frac{f\left(A_{n}\right)-f\left(A_{0}\right)}{g\left(A_{n}\right)-g\left(A_{0}\right)} \leq \prod_{i=1}^{n}\left(\frac{f\left(A_{i}\right)-f\left(A_{i-1}\right)}{g\left(A_{i}\right)-g\left(A_{i-1}\right)}\right)^{\frac{f\left(A_{i}\right)-f\left(A_{i-1}\right)}{f\left(A_{n}\right)-f\left(A_{0}\right)}} .
$$

From these expressions we can obtain inequalities for Stolarsky's $[2,13]$ extended mean value

$$
E_{r, s}(a, b)=\left(\frac{r\left(a^{s}-b^{s}\right)}{s\left(a^{r}-b^{r}\right)}\right)^{\frac{1}{s-r}}
$$

by setting $f(x)=x^{s} / s, g(x)=x^{r} / r, A_{n}=b, A_{0}=a$ and then raising both sides to the power $1 /(s-r)$. For $r s>0$

$$
\begin{aligned}
\left(\frac{b^{s}-u^{s}}{b^{r}-u^{r}}\right)^{\frac{b^{r}-u^{r}}{b^{r}-a^{r}}}\left(\frac{u^{s}-a^{s}}{u^{r}-a^{r}}\right)^{\frac{a^{r}-a^{r}}{b^{r}-a^{r}}} & \leq \frac{b^{s}-a^{s}}{b^{r}-a^{r}} \\
& \leq\left(\frac{b^{s}-u^{s}}{b^{r}-u^{r}}\right)^{\frac{b^{s}-u^{s}}{b^{r}-a^{s}}}\left(\frac{u^{s}-a^{s}}{u^{r}-a^{r}}\right)^{\frac{s^{s}-a^{s}}{b^{s}}}
\end{aligned}
$$

where $a<u<b$.

If $r s<0, f(x)=x^{s} / s$ and $g(x)=x^{r} / r$ are still both increasing functions and we have a similar inequality

$$
\begin{aligned}
\left(\frac{r\left(b^{s}-u^{s}\right)}{s\left(b^{r}-u^{r}\right)}\right)^{\frac{b^{r}-u^{r}}{b^{r}-a^{r}}\left(\frac{r\left(u^{s}-a^{s}\right)}{s\left(u^{r}-a^{r}\right)}\right)^{\frac{u^{r}-a^{r}}{b^{\prime}-a^{r}}}} & \leq \frac{r\left(b^{s}-a^{s}\right)}{s\left(b^{r}-a^{r}\right)} \\
& \leq\left(\frac{r\left(b^{s}-u^{s}\right)}{s\left(b^{r}-u^{r}\right)}\right)^{\frac{b^{s}-u^{s}}{b^{r}-a^{s}}}\left(\frac{r\left(u^{s}-a^{s}\right)}{s\left(u^{r}-a^{r}\right)}\right)^{\frac{u^{s}-a^{s}}{b^{r}-a^{s}}}
\end{aligned}
$$

where it is now necessary to include $r / s$ or else reverse the inequality.

A further application is obtained by setting $f(x)=x$ and $g(x)=\ln x$ above to obtain

$$
\left(\frac{A_{n}-A_{0}}{\ln \left(A_{n}\right)-\ln \left(A_{0}\right)}\right)^{\ln \left(A_{n}\right)-\ln \left(A_{0}\right)} \geq \prod_{i=1}^{n}\left(\frac{A_{i}-A_{i-1}}{\ln \left(A_{i}\right)-\ln \left(A_{i-1}\right)}\right)^{\ln \left(A_{i}\right)-\ln \left(A_{i-1}\right)}
$$

and

$$
\left(\frac{A_{n}-A_{0}}{\ln \left(A_{n}\right)-\ln \left(A_{o}\right)}\right)^{A_{n}-A_{0}} \leq \prod_{i=1}^{n}\left(\frac{A_{i}-A_{i-1}}{\ln \left(A_{i}\right)-\ln \left(A_{i-1}\right)}\right)^{A_{i}-A_{i-1}} .
$$

These two inequalities provide a direct generalization and converse to the main inequality (2) discussed in this paper. 


\section{References}

[1] E. F. Beckenbach and R. Bellman, Inequalities, second ed. (Springer, Berlin, 1965).

[2] P. S. Bullen, D. S. Mitrinović and P. M. Vasić, Means and their inequalities (D. Reidel Publishing Co., Dordrecht, 1988).

[3] F. Burk, "By all means", Amer. Math. Monthly 92 (1985) 50.

[4] B. C. Carlson, "The logarithmic mean", Amer. Math. Monthly 79 (1972) 615-618.

[5] W. E. Diewart, "Superlative index numbers and consistency in aggregation", Econometrika 46 (1978) 883-900.

[6] H. Van Haeringen, "Convex fuzzy sets, unimodal functions, and inequalities for the logarithmic mean", Delft Progress Report 8 (1983) 173-179.

[7] G. H. Hardy, J. E. Littlewood and G. Polya, Inequalities (Cambridge University Press, Great Britain, 1967).

[8] J. Heidel and J. Maloney, “An analysis of a fractal Michaelis-Menton equation”, J. Austral. Math. Soc. Ser. B, 41 (2000) 410-422.

[9] G. Lorenzen, “Log-ratios and the logarithmic mean", Stat. Papers 30 (1989) 61-75.

[10] D. S. Mitrinović, Analytic inequalities (Springer, Berlin, 1970).

[11] D. S. Mitrinović, J. E. Pečarić and A. M. Fink, Classical and new inequalities in analysis (Kluwer Academic Publishers, Dordrecht, 1993).

[12] D. Ruthing, “Eine Allgemeine Logarithmische Ungleichung”, El. Math. 41 (1996) 14-16.

[13] K. B. Stolarsky, "The power and generalized logarithmic means", Amer. Math. Monthly 87 (1980) 545-548. 